\title{
The pharmacist survival guide: COVID-19 workflow modifications and crisis management in the surgical setting
}

\author{
Jawad N. Saleh ${ }^{1} \mathbb{0}$ \\ Accepted: 21 November 2021 / Published online: 9 December 2021 \\ (c) The Author(s), under exclusive licence to Springer Nature Switzerland AG 2021
}

\section{Background}

Hospital pharmacies have been faced with many challenges during the coronavirus disease 2019 (COVID-19) pandemic. Multiple disciplines comprising administration, critical care/ anesthesia, medicine, nursing, infectious diseases, infection prevention, engineering, informatics, respiratory therapy, and pharmacy worked around the clock to create cohesive and effective intensive care units (ICUs) for these patients.

Our institution is a 220-bed hospital in New York City that specializes in orthopedic surgery and the treatment of rheumatological conditions. We have an orthopedic specialty care unit, step down unit (SDU), and three post-anesthesia care units (PACUs). In preparation to take on critically ill intubated patients, both the operating rooms (ORs) and PACUs were both successfully converted into a fully functional medical intensive care unit (MICU).

The purpose of this survival guide is to describe in detail the hurdles and challenges, from a pharmacist's perspective, and to provide a guide for pharmacists on how to prepare and succeed whenever faced with this tremendous challenge. The guide provides a step-by-step approach in regard to room configurations, equipment handling, staffing, and medication management. The recommendations go into ICU conversions, automatic dispensing cabinet (ADC) placements, staff preservation, pump insufficiencies, drug shortages, medication bundling, and employee wellness as well as different approaches to minimize contamination and exposure.

Jawad N. Saleh

Salehj@hss.edu

1 Pharmacy Department, Clinical Services, The Hospital for Special Surgery, 535 E70th St., New York, NY 10021, USA

\section{Methods}

\section{Room configurations}

Negative pressure has become a common term in this global crisis. Engineering was an integral part of the ICU conversion process in our institution, with success in the conversion of isolation inpatient rooms, ORs, and open PACU suites to negative-pressure areas. They are called negative-pressure rooms because the air pressure inside the room is lower than the air pressure outside the room. This means that when the door is opened, potentially contaminated air or other dangerous particles from inside the room will not flow outside into non-contaminated areas. The importance of understanding where the negative-pressure areas exist is vital to the pharmacy workflow.

ORs in our institution were converted from positive- to negative-pressure rooms to protect staff (Fig. 1). These rooms had two different doors, located on opposite ends of the room. This allowed access to multiple corridors-which made them optimal when entering and exiting a contaminated area. You enter from the clean corridor and exit into the dirty corridor on the other side. All air was converted to $100 \%$ exhaust; fresh air came from outside.

PACUs or open area suites were also converted to negative-pressure areas (Fig. 2). Don and doff bay areas were converted to positive pressure to help minimize contamination. Depending on the room type and unit set-up, an anteroom chamber may be necessary. Anteroom chambers should be strategically placed outside of negative-pressure rooms if a doff bay area is not feasible and/or if the entire unit was not converted to a negative-pressure COVID-19 unit.

Understanding the workflow of donning and doffing and complying with the United States Pharmacopeia (USP) standards was crucial in minimizing contamination and preserving the safety of our healthcare staff. These areas differed depending on the configuration of each unit. In the 


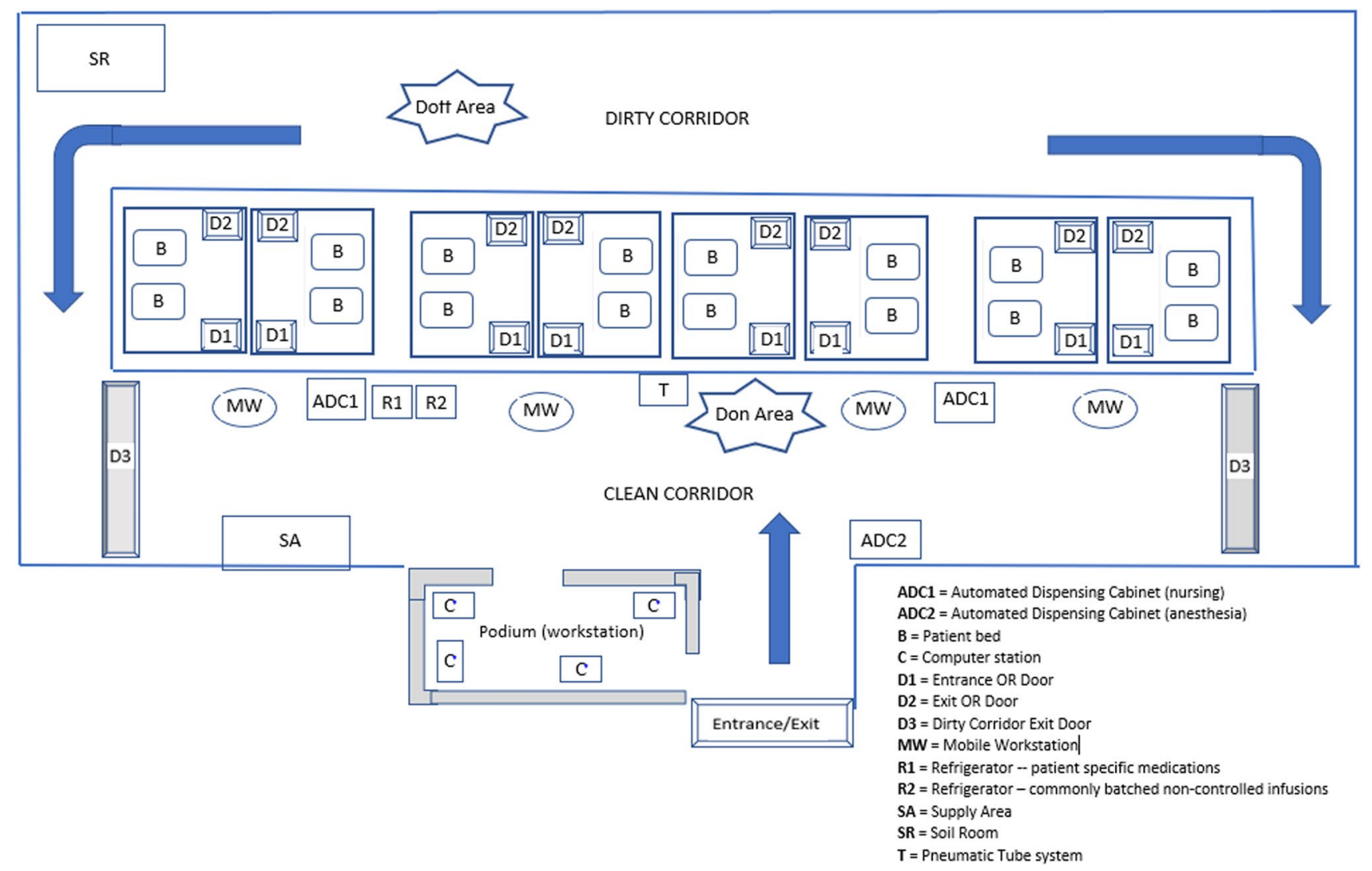

Fig. 1 Operating room conversion to COVID-19 medical intensive care unit. COVID-19 coronavirus disease 2019

OR conversion, a clean corridor existed in which the donning occurred. This was the area in which the workstation also existed and where clinical pharmacists, nurses, and physicians worked. Once the healthcare provider entered the room, they were not allowed to reemerge through those doors. They would exit from doors on the opposite side of the room to what was assigned as the "dirty" corridor. This was the area in which the staff would doff. This differed from the PACU conversion, which resembled an open suite containing COVID-19 patients. Construction was needed to create walls to isolate the podium area with windows allowing visibility to patient care. Two small areas existed between this closed-off area and the actual unit (dirty area). This is where the team members would don/doff when exiting the negative-pressure unit. This was a positive-pressure area to minimize contamination amongst the healthcare team.

Creating and appointing runners in these units will also help with medication workflow. "Runners" are nurses in the clean area that are assisting their fellow nurses who are working in the COVID-19 rooms/unit treating these patients. The runner will obtain any medications needed from the pyxis machine, patient specific drawer/bag, or refrigerator and deliver it directly to the nurse providing direct patient care (through anteroom, negative-pressure door). This helps maintain a designated clean area that allows for a safer pharmacy refill/medication delivery process and minimizes both contamination and exposure. Without runners, there is increased risk for contamination and delays in patient care.

\section{Equipment}

The need for ADCs and their placements in these units must be discussed with the pharmacy department prior to construction. It is best that these machines be strategically placed in clean areas. Once the area is agreed upon by pharmacy, nursing, and the critical care team, contact engineering to place adequate electrical outlets. Follow-up with the network team to facilitate the set-up. The goal here is to prevent pharmacy staff exposure as well as minimize medication contamination. Again, the placements differ in locations depending on the unit configuration. Our institution carried two different types of ADCs. One was specifically for the anesthesia team located predominantly in the ORs, and the other was used by the rest of the institution. Consider disabling the biometric fingerprint requirements and utilizing username and password (if state regulations permit). This will prevent removal of gloves to sign into the machine. Appropriate disinfectant should be placed by 


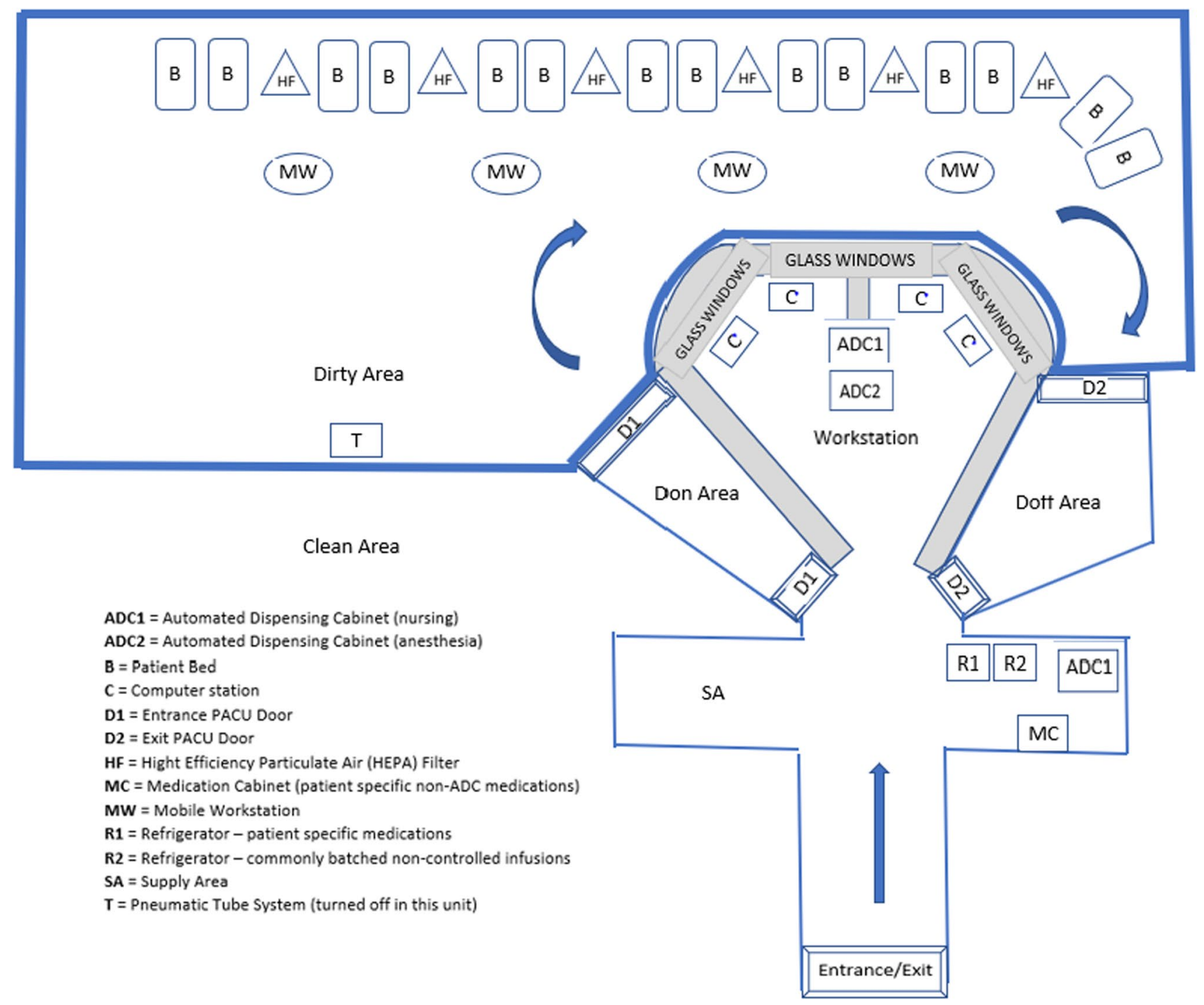

Fig. 2 Post-anesthesia care unit (PACU) conversion to COVID-19 medical intensive care unit. COVID-19 coronavirus disease 2019

each machine and utilized before and after use. The other recommendation would be to temporarily increase medication par levels to decrease both the frequency of restocking and the traffic to ADCs. Continuously run utilization reports weekly and remove slow-moving medications to help increase capacity. Lastly, ADCs moved to different locations should be renamed to prevent confusion.

ADC education and access may help with several workflow inconveniences. Different disciplines will step into roles that they are not accustomed to. ADC access and education must be done properly to prevent workflow discrepancies. Obtain a staff list from the manager of that specific unit and begin granting access immediately while providing in-services regularly. Based on the acuity of patients, we increased the amount of medications that can be overridden in an emergency without having an order in place. Common
ADC "override" medications included vasopressor infusions (norepinephrine, phenylephrine, epinephrine), rapid sequence intubation (RSI) kits, and neuromuscular blocking agents (NMBAs) (cisatracurium and rocuronium). RSI kits were created for override and included succinylcholine, rocuronium, propofol, etomidate, and ephedrine.

In addition to the ADCs, two medication refrigerators (regardless of unit) would be ideal for this patient population: one refrigerator for patient-specific medications and the other for storing the most common non-controlled infusions batched by pharmacy (require prior pyxis removal to track inventory).

Non-ADC medications were delivered by pharmacy, and placement differed based on unit. In the OR conversion unit, these medications were placed in a patient-specific bag that hung outside of the patient room. In the PACU conversion, 
we had a cart with patient-specific drawers specifically for these medications that was placed in a small clean room alongside the refrigerators.

Although the PACU conversion and open area suite designs were converted to negative-pressure units, the size of the open area may require additional filtration. Utilization of high-efficiency particulate air (HEPA) exhaust fans was necessary, with additional HEPA filters added when necessary.

Mobile workstations, laptops, and tablets were also a necessity in optimizing workflow and minimizing contamination. This was due to the reduction in entering and exiting the unit. Mobile workstations can include a combination of a computer and several drawers for storage use. These act like point-of-care carts and were used in a variety of different ways, with their primary importance being inputting patient data.

\section{Staffing}

Due to the complexity of this disease, staffing has remained a challenge for all healthcare personnel. Training must occur with both non-clinical and non-critical care certified/trained pharmacists, with proper resources, such as COVID-19-specific lecture series, routine clinical case/practice meetings, and critical care materials from professional societies. A buddy system should be established between clinical pharmacists and staff pharmacists.

In efforts to avoid losing staff by preventing exposure and contamination, pharmacist schedules were all adjusted accordingly. Although the halting of elective surgeries decreased overall patient volume, the increase in complexity and acuity of the COVID-19 population demanded around-the-clock care. At our institution, we have both staff and clinical pharmacists with different job descriptions and workflows. The clinical pharmacists are unit based and work on inpatient floors dealing with direct patient care. Staff pharmacists cover daily operations such as order verification, compounding, and inventory. All pharmacists work 5 days a week, with 8-h work shifts and rotating weekends. In order to adjust to the surge, staff members were grouped into three separate teams, which worked separately from one another (Table 1).

Cardiac arrests increased dramatically, with multiple emergencies occurring simultaneously [1]. Ensure you have proper staffing, and make appropriate efforts to always have a clinical pharmacist on site to help manage emergencies. Due to the pandemic, pharmacy code responsibilities may change significantly in efforts to minimize contamination. Communicate with the healthcare team in regard to clean areas and where pharmacists will stand during these emergencies. Have a walk-through with your front-line pharmacists in different units and explain where they are to position themselves during a cardiac arrest. Try and limit the number of people inside the room (e.g. maximum of five). Emergency carts should almost exclusively be located in clean areas, with each medication put in a plastic bag to minimize contamination and salvage some unused medications in short supply. Recommend pharmacists and code carts remain outside of the room (OR ICU) or outside of open unit

Table 1 Staffing methods

\begin{tabular}{|c|c|c|c|c|}
\hline Teams & Location & Shift & Hours & Work days \\
\hline Team A & On-site & Day & $12.5 \mathrm{~h}$ & 2 on and 3 off, then 2 on and 2 off, then 3 on and 2 off \\
\hline Team B & On-site & Evening & $12.5 \mathrm{~h}$ & 2 on and 3 off, then 2 on and 2 off, then 3 on and 2 off \\
\hline Clinical & On-site & Day + evening & $12.5 \mathrm{~h}$ & Expanded coverage (around the clock if possible) \\
\hline \multicolumn{5}{|c|}{$\begin{array}{c}\text { If one team is compromised due to exposure, recruit team from below } \\
\qquad \downarrow\end{array}$} \\
\hline Team C & Home & Day + evening & $12.5 \mathrm{~h}$ & 2 on and 3 off, then 2 on and 2 off, then 3 on and 2 off \\
\hline Team D & Off & Variable & Variable & Variable-emergency mode to fill shifts \\
\hline
\end{tabular}

If one on-site team gets infected, the at-home team (team C) comes in

If understaffing continues, you can attempt the creation of a new team consisting of per-diems, part-timers, residents, and interns who have not been on site (team D)

Pharmacists should social distance and work in workstations at a set distance from one another. Examples of this include the IV pharmacist remaining in the IV room for the entire shift and the staggering of lunch breaks (with maximum number of pharmacists in the break room monitored regularly)

Minimal overlap-hand-off communication should be done via email or over the phone (disinfect workstation before and after shift)

Two on and 3 off, then 2 on and 2 off, then 3 on and 2 off-gives the employee consistent days off (childcare)

Seven days on and 7 days off is an option, but a big downside is that 7 consecutive days may cause mental fatigue

The clinical team should social distance from the operational staff to avoid exposure from one another (especially if clinical team is rounding on the COVID unit)

COVID coronavirus disease, $I V$ intravenous 
(PACU ICU). "Emergency bags" were created and left in each of the OR ICU rooms, and several bags were placed in the PACU converted unit in an effort to minimize emergency tray contamination The bag contents included epinephrine $(2 \times 1 \mathrm{mg} / 10 \mathrm{~mL}$ prefilled syringes), norepinephrine $(2 \times$ $4 \mathrm{mg} / 4 \mathrm{~mL}$ vials $)$, phenylephrine $(2 \times 10 \mathrm{mg} / 1 \mathrm{~mL}$ vials $)$, vasopressin $(2 \times 20$ units $/ 1 \mathrm{~mL}$ vials $)$, atropine $(1 \mathrm{mg} / 10$ $\mathrm{mL}$ prefilled syringe $)$, and calcium chloride $(2 \times 1 \mathrm{~g} / 10 \mathrm{~mL}$ prefilled syringes). Communication with the healthcare team on pharmacy's new role in these situations is needed (help with making infusions, dosing, and contraindications, etc.).

\section{Medications}

The process of medication returns varies from institution to institution. If done incorrectly, it may result in pharmacy contamination. To reduce contamination, medications that are likely contaminated may be returned to the pharmacy after the proper steps are taken. The nurse wipes down the medication with disinfectant, and the medication is then placed in a paper bag. Plastic bags have a higher surface viral load and were not used for this purpose [2]. The bags should be sprayed down/cleaned before and after use, labeled with incident date, and then placed in a "return" bin for pharmacy pick up. Pharmacy picks up the bag, disinfects the bag again, and places the bag in the appropriate storage area for sequestered medications in the pharmacy department. Medications are sequestered for $72 \mathrm{~h}$, unless it is a shortage item, for which disinfection will take place. The virus is more viable on plastics and steel than it is on copper and cardboard [2]. However, it should be noted that the replication times can vary depending on a number of factors such as temperature, humidity, ventilation, and the amount of virus deposited [3].

Whether or not to turn off the pneumatic tube system was discussed extensively. Depending on location and positioning of the pneumatic tube in that specified unit, some tube stations were turned off (dirty area), while others were kept on (clean area). Utilizing tubes outside of isolation wings helps avoid direct contact with the isolation unit. Pneumatic tubes outside of these units should ideally still be in close proximity to the patients, and the runners will take these medications to the nurse in the room or unit. If runners are not in place, allow inbound tubes to isolation units, but no outbound. Utilize central supply systems to clean and retrieve pneumatic tubes throughout the day and return to the pharmacy. For areas in which the pneumatic tube system is functioning, continue to utilize the tube system as normal and promote hand hygiene. Cleaning of the tubes with proper disinfectant, both externally and internally should be conducted on a routine basis at the beginning of every shift and when receiving or returning to other stations (may affect cleaning supplies that could be crucial for other areas). If you decide to shut down the pneumatic system, please consider that while you may reduce contamination by reducing the number of used/contaminated medications and lab specimens being sent through the tube system, you may increase staff contamination as a result of the increased need for delivery to/from these units.

Pump insufficiency has become a common issue during the pandemic. This patient population requires multiple infusions, which may lead to several pumps being used on the same patient simultaneously. Search units in which pumps are not being used and retrieve them (e.g. interventional radiology, perioperative areas, outpatient settings) for use in the ICU. Consider using your patient-controlled analgesia (PCA) pumps for your pain infusions in order to alleviate some of the other smart pumps for other infusions. Another option, although not optimal, is to utilize gravity flow infusions for medications that can be safely managed without the alerts built into the smart pumps [intravenous (IV) hydration, some IV antibiotics]. Utilize a gravity flow rate drip chart when appropriate (Table 2) [4]. Maximize oral formulations via nasogastric (NG)/percutaneous endoscopic gastronomy (PEG) tube and/or intermittent IV pushes in attempting to wean patients off of the infusions.

Updates to the smart pump library are crucial in this crisis and should be done immediately and frequently to minimize error. Updates to the pump library should reflect the order set created. Nursing education on new libraries or updates to existing library should be communicated as they happen. It is recommended no more than two different concentrations for an infusion be created. Clinicians should attempt to use the more concentrated infusion option for this patient population due to high dose demand and fluid sensitivity

Positioning infusion pumps outside of COVID-19 patients' rooms can help preserve personal protective equipment (PPE) and reduce the frequency of exposure that nurses would ordinarily experience by going into patients' rooms to manage infusions. This should only be done for isolated single-patient rooms. Hallway placement of pumps should be discouraged in multi-occupant rooms and may not be practical in certain unit configurations. Examine the workspace prior to making these decisions and ensure sufficient power plugs are available for all pumps held in the hallways. Pumps outside of rooms seem to work best with central lines when low flow through extension tubing is needed and arterial line blood pressure monitoring is preferred.

Consider cosign procedures for high-risk medications and modifying bar-code scanning in the COVID-19 units. Historically, the independent double checks (IDC) provide a safeguard at the point of administering high alert medications, but in this patient population, a recommendation to modify this safeguard (risk/benefit) may be needed. To support workflow of medication administration practices for COVID-19 patients in isolation, recommendations for 
Table 2 Gravity flow rate drip chart

\begin{tabular}{|c|c|c|c|c|}
\hline Flow rate $(\mathrm{mL} / \mathrm{h})$ & 10 drops $=1 \mathrm{~mL}(\mathrm{drops} / \mathrm{min})$ & 15 drops $=1 \mathrm{~mL}($ drops $/ \mathrm{min})$ & 20 drops $=1 \mathrm{~mL}(\mathrm{drops} / \mathrm{min})$ & $\begin{array}{l}60 \text { drops }=1 \\
\mathrm{~mL} \text { (drops/ } \\
\mathrm{min} \text { ) }\end{array}$ \\
\hline 10 & 2 & 2 & 3 & 10 \\
\hline 25 & 4 & 6 & 8 & 25 \\
\hline 50 & 8 & 12 & 17 & 50 \\
\hline 75 & 12 & 19 & 25 & 75 \\
\hline 100 & 17 & 25 & 33 & 100 \\
\hline 125 & 21 & 31 & 42 & 125 \\
\hline 150 & 25 & 37 & 50 & 150 \\
\hline 200 & 33 & 50 & 67 & 200 \\
\hline 250 & 42 & 62 & 83 & 250 \\
\hline 500 & 83 & 125 & 167 & 500 \\
\hline 1,000 & 167 & 250 & 333 & 1000 \\
\hline
\end{tabular}

Confirm tubing set drip rate on set package, i.e. $10,15,20$, or 60 drops $/ \mathrm{mL}$

Recommend that all gravity infusion bags be tapped for additional flow confirmation

Alterations of bag height distance to patient will affect flow rate

practice changes around second nurse verification badge/ patient ID scanning should be considered. Bar Code Medication Administration (BCMA) considerations should be made depending on whether the pumps are located inside or outside of the patient rooms.

Drug shortages have become a huge dilemma for many hospital pharmacies during the COVID-19 crisis [5, 6]. Try and recommend keeping up-to-date inventory, with projected usage of high-demand medications on a weekly basis. Ensure that you share any inventory changes with the frontline pharmacists and with administration daily. It is crucial to create an "alternative" drug list. This is where pharmacy can play a critical role. This is based on drug or class of drugs categorized as option 1, 2, and 3. It is strongly suggested to work with a physician champion nominated by that specialty to be the point person with "alternative" decision making (e.g. option 1: fentanyl; if short, $\rightarrow$ option 2: hydromorphone; if short, $\rightarrow$ option 3: morphine). With assistance from the physician champion, pharmacy will calculate the average drug supply needed per patient to gauge inventory and what needs to be stocked. Avoid multiple requests from different individuals. This becomes problematic during drug shortages in a crisis. You will end up over-ordering, burning your budget and taking up much needed space for medications you will never use. Speaking to the chief of that department about a champion nomination should be done immediately. For other requests, defer the requestor to the champion. Consider different concentrations, doses, and even routes in a shortage. IV room pharmacists may need additional staffing as compounding will increase during a drug shortage (batching used to minimize drug waste and conserve PPE). Also, continue to seek 503B outsourcing facility flexibility to help ensure they can meet your institution's medication needs. Assigning someone to work on beyond use dates (BUD) of compounded medications and creating a list that staff can refer to will be very helpful. Extended BUD are important in these times, especially for workflow management. Cost savings and initiatives are typically put on hold as the surge gets worse. Conserving injectables and metereddose inhalers (MDIs) for COVID-19 patients and maximizing other formulations for non-COVID patients will help allocate medications accordingly.

Order creations/modifications will increase as these infusions begin to surface. The informatics pharmacist will get busy very fast with the addition of new medications, doses, concentrations, infusions, and rounding tools (to help pharmacists without critical care experience) and creation of best practice safety alerts. COVID-19 order sets should be created to help prescribers order effectively and safely. These include the sedatives, analgesics, NMBAs, and vasopressor infusions. Try to enhance pharmacy privileges for both clinical and operational staff, as this will greatly reduce the number of phone calls, text messages, and pages to an overwhelmed medical team. Some of these privileges include medication time changes, expansion of automatic substitutions list, infusion bag volume changes (if same concentration based on dose), and addition of instructional notes in the actual order itself to help clarify orders that may cause confusion to the healthcare team.

Bundling medications has become a common practice in which pharmacy has played an integral part. In an effort to help limit the frequency of nurse-to-patient contact, consolidation of medication times (bundling together) helped minimize contamination as well as conserve PPE. Consider 
utilizing three times a day or twice daily dosing rather than every 8 or $12 \mathrm{~h}$, as clinically appropriate, to align administration times. We switched all administration times to 10 a.m. and 10 p.m. When possible, try to time other medications around the medications that are time sensitive as well as those that involve drug-drug interactions. Medications requiring multiple administrations are discouraged unless clinically necessary. For non-COVID-19 patients, continue to switch medications from IV to oral/NG tube as appropriate to help extend injectable supply.

Evidence continues to grow when it comes to COVID19 medication management. Creating a simplified protocol defining the eligibility criteria for different therapies (including vaccines) is strongly encouraged.

\section{Health and well-being}

It is widely known that our surrounding environment and experiences can lead to post-traumatic stress disorder, directly impacting our health and well-being [7, 8]. In these hard times, support from staff and administration plays an integral part in helping colleagues cope with the current environment. Continue to advertise the wellness resources that exist and how employees can access them.

Support methods include daily live streams by administration, motivational video snippets, uplifting messages (posters, quotes), children's artwork, wellness emails (coping methods), staff recognition, and senior leaderships participation in inter-disciplinary rounds. Mental health support resources should be readily available to the entire staff.

\section{Conclusion}

This survival guide offers a wealth of detail from the COVID-19 pandemic to ultimately help hospitals cope with the patient surges faced nationwide. The goal was to provide fellow pharmacists with key points to help overcome the struggles of a surge and alleviate some of the workflow anxiety associated with a pandemic. The article goes into great depth with every component of the ICU conversionengineering, information technology, workflow design, and administrative issues. The emphasis on overall medication management during a pandemic was crucial in minimizing contamination and avoiding waste. Bundling or consolidating medication times as well as strict medication return techniques were key strategies in limiting staff exposure and reducing contamination. This guide can be extremely beneficial when preparing for an overwhelming surge and should be utilized when dealing with any pandemic. A formulated plan needs to be made prior to the surge, and preparation in advance is crucial to succeed.
A limitation to this guide is that the institution being referenced throughout the guide specializes in orthopedic surgery, and other general hospitals may not be able to implement some of the recommendations' structure and design. Additionally, institutions may utilize different equipment (ADCs, pumps, IT, pneumatic tubes) and may not benefit fully from some of the points mentioned.

Acknowledgements The author would like to acknowledge critical care anesthesiologists Sean Garvin MD, Meghan Kirksey MD, PhD, Jemiel Nejim MD, Stavros Memtsoudis MD, PhD, MBA, and Elaine Yang MD for the sacrifices they made during this pandemic. Your dedication, commitment, and courage deserve the deepest gratitude and admiration. Your service to patients saved countless lives and made such a difference in getting through this crisis. I would also like to acknowledge Nicole Oliva, PharmD, BCPS Senior Director of Pharmacy, for working diligently on operational workflow, medication management, and overall support during this crisis. Lastly, I would like to say how proud I am to have worked side by side with some of the most amazing and courageous individuals one can ask for in these dark times. I would also like to say how honored and privileged I am to work for an organization with a culture and ethical drive that celebrates the value of its employees. A special thank you to all of the front-line healthcare workers around the world and the unrecognized pharmacists who remain in the shadows. Thank you.

\section{Declarations}

Funding No funding has been received for the preparation of this article.

Conflicts of interest None of the authors have conflicts of interest to disclose.

Ethical approval Not applicable.

Consent to participate Not applicable.

Consent to publish Not applicable.

Availability of data and materials Not applicable.

Author contributions JNS drafted and revised the manuscript.

\section{References}

1. Miles J, Mejia M, Rios S, et al. Characteristics and outcomes of in hospital cardiac arrest events during the COVID-19 Pandemic. Circ Cardiovasc Qual Outcomes. 2020;13(11):222.

2. Van Doremalen N, Bushmaker T, Morris DH, et al. Aerosol and surface stability of SARS-CoV-2 as compared with SARS-CoV-1. N Engl J Med. 2020;382(16):1564-7.

3. Chin Alex WH, Chu Julie TS, Perera Mahen RA, et al. Stability of SARS-COV-2 in different environmental conditions. Lancet Microbe. 2020;1(1):e10.

4. Morris DG. Intravenous calculations. In: Morris DG, editor. Calculate with confidence. 6th ed. St. Louis: Elsevier; 2018. p. 536-602.

5. ASHP Drug Shortages: http://www.ashp.org/drug-shortages/curre nt-shortages. Accessed 12 Apr 2020. 
6. FDA Drug Shortages: https://www.fda.gov/drugs/drug-safety-andavailability/drug-shortages. Accessed 30 Mar 2020.

7. Boyraz G, Legros DN, Tigershtrom A. COVID-19 and traumatic stress: the role of perceived vulnerability, COVID-19-related worries, and social isolation. J Anxiety Disord. 2020;76:102307.
8. Shanafelt T, Ripp J, Trockel M. Understanding and addressing sources of anxiety among health care professionals during the COVID-19 pandemic. JAMA. 2020;323(21):2133-4. 